\title{
Dúvidas na indicação cirúrgica da cerclagem do tronco pulmonar
}

FRANCISCO GREGORI Jr. *, ROBERTO TAKEDA*, S. S. SILVA*, L. FAÇANHA**, I. RIBEIRO**, M. FABIA$\mathrm{NI}^{\star \star \star}$, G. GALVĀO ${ }^{\star \star \star}$, F. G. ROCHA ${ }^{\star \star \star}$, L. E. GOIS ${ }^{\star \star \star}$, W. AQUINO**, J. E. SIQUEIRA*, P. KRELING*, L. C. MIKITA***

GREGORI Jr., F.; TAKEDA, R.; SILVA, S. S.; FAÇANHA, L.; RIBEIRO, I.; FABIANI, M.; GALVĀO, G.; ROCHA, F. G.; GOIS, L. E.; AQUINO, W.; SIQUEIRA, J. E.; KRELING, P.; MIKITA, L. C. - Dúvidas na indicaçāo cirúrgica da cerclagem do tronco pulmonar. Rev. Bras. Cir. Cardiovasc., 1(2):1-8, 1986.

RESUMO: No periodo de fevereiro de 1979 a março de 1986 foram submetidos a cerclagem do TP 40 pacientes portadores de cardiopatias congênitas com hiperfluxo pulmonar, insuficiência cardiaca e hipodesenvolvimento ponderal. Vinte e três pacientes eram do sexo masculino e 17 do feminino com idade média de 6 meses (20 dias a 17 meses). Foram as seguintes as malformaçōes: CIV (22 casos), AVC total (6), DVSVD (2), TA (2), TGVB (3), AT (2), VU (1) e cardiopatia complexa (2 casos). A cerclagem foi realizada com controle de pressão do TP de tal forma a reduzíla de $50 \%$ nos acianóticos e de $40 \%$ nos cianóticos. Dois pacientes $(5 \%)$ faleceram no pós-operatório imediato, sendo um em bloqueio $A-V$ total (AVC, $1,7 \mathrm{~kg}, 20$ dias de idade) e outro em septicemia, 20 dias após a cirurgia (CIV). Um óbito tardio ocorreu por broncopneumonia. Os demais pacientes evoluíram satisfatoriamente, com resolução da ICC e ganho de peso em média $400 \mathrm{~g}$ por mês (peso pré: média $4 \mathrm{~kg}$, peso pós: média $7,5 \mathrm{~kg}$ ). Treze pacientes foram reestudados, hemodinamicamente, em média 21 meses após a cerclagem. A relação FP/FS reduziu de 2,7 para 0,92 , ao mesmo tempo em que houve diminuição também na relação PSAP/PSAo de 0,73 para 0,28 . Nestes pacientes houve uma melhora clínica observada pela diminuição do déficit ponderal de 38 por cento, para 20 por cento. Treze pacientes foram submetidos a correção total em média 21 meses após a cerclagem, com dois óbitos hospitalares, sendo um imediato e outro por intoxicação digitálica, 1 mês após a cirurgia. Analisando-se, separadamente, os 6 casos de AVCT, em todos predominava o shunt e nāo o refluxo valvar. Houve um óbito imediato e 5 pacientes foram reestudados hemodinamicamente. Observou-se redução importante nas pressōes pulmonares e no fluxo arterial pulmonar. Estes pacientes foram reoperados para correção total, com boa evolução.

DESCRITORES: cerclagem do tronco pulmonar.

\section{INTRODUÇÃO}

Desde a introdução da cerclagem do tronco pulmonar, em $1951^{5}$, uma variedade de lesōes cardíacas congênitas com aumento do fluxo arterial pulmonar tem sido, efetivamente, tratada.
Com o aperfeiçoamento dos métodos de circulação extracorpórea, excelentes resultados têm sido obtidos com o tratamento cirúrgico corretivo dos pacientes com baixo peso corpóreo. Este fato, somado aos resultados controversos da cerclagem do tronco pulmonar, observados na literatura, tem

Trabalho realizado na Fundação Universidade Estadual de Londrina, Hospital Evangélico de Londrina e Santa Casa de Londrina, Londrina, PR, Brasil. 
GREGORI Jr., F.; TAKEDA, R.; FAÇANHA, L.; RIBEIRO, I.; FABIANI, M.: GALVÃO, G.; ROCHA, F. G.; GÖIS, L. E.; AQUINO W.; SIQUEIRA, J. E.; KRELING, P.; MIKITA, L. C. - Dúvidas na indicação cirúrgica da cerclagem do tronco pulmonar. Rev. Bras. Cir. Cardiovasc., 1(2):1-8, 1986.

levado a maioria dos cirurgiōes a preferir o método corretivo como primeira instância.

No Serviço de cirurgia cardíaca de Londrina, desde 1979, temos indicado a cirurgia de cerclagem do tronco pulmonar aos pacientes com cardiopatia congênita e hipodesenvolvimento pônderoestatural, reservando o método corretivo com circulação extracorpórea somente aos casos com peso corpóreo acima de $6 \mathrm{~kg}$ e idade superior a 6 meses. O objetivo deste estudo é apresentar os resultados pós-operatórios dos pacientes submetidos a este tipo de procedimento cirúrgico.

\section{CASUÍSTICA E MÉTODOS}

No período de fevereiro de 1979 a março de 1986 , foram submetidos a cerclagem do tronco pulmonar 40 pacientes portadores de cardiopatias congênitas com hiperfluxo pulmonar, hipodesenvolvimento pôndero-estatural e insuficiência cardíaca ae difícil resolução clínica. As indicaçōes para a cerclagem foram o controle da insuficiência e a prevençăo do estabelecimento de doença vascular pulmonar.

Vinte e três pacientes eram do sexo masculino e 17 , do sexo feminino, com idade mínima de 20 dias e máxima de 18 meses (média 5 meses).

Pacientes com idade acima de 6 meses foram submetidos a cerclagem, ou por apresentarem cardiopatias complexas, ou por estarem em estado clínico geral bastante comprometido.

O peso médio pré-operatório dos pacientes foi de $4 \mathrm{~kg}$, sendo o mínimo de $1,8 \mathrm{~kg}$ e o máximo de $8 \mathrm{~kg}$.

As patologias encontradas foram: comunicação interventricular isolada (CIV) (22 casos), atrioventricularis communis totalis(AVCT) (6 casos). transposiçăo das grandes artérias (TGV) ( 3 casos), dupla via de saída do ventrículo direito (DVSVD) (2 casos), atresia tricúspide (AT) (2 casos), truncus arteriosus (TA) (2 casos), ventrículo único (VU) (1) e cardiopatia congênita complexa (CCC) (2 casos).

Em 36 pacientes a via de acesso cirúrgica utilizada foi a toracotomia anterior no terceiro espaço intercostal esquerdo, com a posição do paciente discretamente lateralizada. Em 2 pacientes foi utilizada esternotomia mediana e, em 2, esternotomia transversa.
Para a constrição do tronco pulmonar, empregamos fita cardiaca comum. Experiência prévia tem mostrado que crianças com shunt esquerda - direita com defeitos cardíacos não complexos podem ter um fluxo pulmonar adequadamente diminuído utilizando-se fita com circunferência de $20 \mathrm{~mm}$ mais $1 \mathrm{~mm}$ por quilograma de peso corpóreo. Em pacientes com defeitos complexos ou shunt bidireçional, a circunferência da fita pode ser calculada na base de $24 \mathrm{~mm}$ e $1 \mathrm{~mm}$ por quilograma de peso corpóreo $^{8}$.

Utilizamos, em adicional, como parâmetro muito importante para o controle do grau de constrição, a monitorização da pressāo hidrostática do tronco pulmonar, de modo a diminuir 50 por cento nos acianóticos e 40 por cento nos cianóticos.

O aparecimento de cianose e diminuição da freqüência cardiaca acima de 20 por cento da inicial consideramos sinais indicativos de cerclagem inadequada, a qual deverá ser desfeita e colocação de fita com circunferência um a dois milimetros maior.

Terminado 0 ato cirúrgico, os pacientes foram encamihados à sala de recuperação, extubados. Dificilmente, houve necessidade de mantê-los no ventilador $e$, quando isto aconteceu, foi indício, em geral, de mau resultado pós-operatório.

Os pacientes foram analisados no pós-operatório tardio (tempo médio 3 anos), observando-se a evolução da insuficiência cardíaca e do desenvolvimento ponderal. A evolução ponderal pós-operatória dos pacientes foi comparada com a dos pacientes com shunt, segundo os estudos de SILVA ${ }^{6}$ (1980).

Treze pacientes foram estudados, hemodinamicamente, no pós-operatório, analizando-se, com especial atenção, a pressão em tronco pulmonar e a relação fluxo arterial pulmonar-fluxo arterial sistêmico. Destes, 5 eram pacientes portadores de AVC total, com predomínio de shunt mais do que o refluxo valvar.

\section{RESULTADOS}

Dois pacientes $(5 \%)$ faleceram no pós-operatório imediato. Um deles tratava-se de paciente com diagnóstico de atrioventricularis communis totalis com peso corpóreo de 1,7 quilograma e 20 dias de idade, evoluindo muito bem nos primeiros 
GREGORI Jr., F.; TAKEDA, R.; FAÇANHA, L.; RIBEIRO, I.; FABIANI, M.; GALVĀO, G.; ROCHA, F. G.; GÓIS, L. E.; AQUINO, W.: SIQUEIRA, J. E.; KRELING, P.; MIKITA, L. C. - Dúvidas na indicaçāo cirúrgica da cerclagem do tronco pulmonar. Rev. Bras. Cir. Cardiovasc., 1(2):1-8, 1986.

TABELA 1

CORRELAÇÃO ENTRE O DÉFICIT PONDERAL E VARIAÇÕES HEMODINAMICAS PRÉ E POSOPERATORIAS NOS PACIENTES SUBMETIDOS A CERCLAGEM DA ARTERIA PULMONAR

\begin{tabular}{|c|c|c|c|c|c|c|c|c|}
\hline \multirow[t]{2}{*}{ IDENT. } & \multirow[t]{2}{*}{ DIAG. } & \multirow{2}{*}{$\frac{\text { T.P.O. }}{\text { (MESES) }}$} & \multirow{2}{*}{$\frac{D E F}{P R E}$} & \multirow{2}{*}{$\frac{\text { POND. (\%) }}{\text { POS }}$} & \multicolumn{2}{|c|}{$F P / F S$} & \multicolumn{2}{|c|}{ PSAPIPSAO } \\
\hline & & & & & PRÉ & PÓS & PRÉ & POS \\
\hline $1-V D L$ & AVC & 18 & 43 & 32 & 1.90 & 0.32 & 0.92 & 0.29 \\
\hline $2-\operatorname{ICS}$ & CIV & 13 & 31 & 14 & 1.82 & 0.82 & 0.43 & 0.25 \\
\hline $3-$ IVS & AVC & 9 & 24 & 14 & 4.10 & 1.10 & 0.56 & 0.23 \\
\hline $4-E M C$ & $\mathrm{ClA}+\mathrm{CIV}$ & 15 & 46 & 20 & 5.00 & 0.69 & 0.76 & - \\
\hline $5-F W P$ & CIV & 38 & 43 & 27 & 3.00 & 0.79 & 0.71 & 0.25 \\
\hline $6-S A O$ & $\mathrm{CIA}+\mathrm{CIV}$ & 27 & 34 & 3 & 1.30 & 1.48 & 0.97 & 0.15 \\
\hline $7-A A$ & DVSVD & 23 & 52 & 36 & 1.19 & - & 1.00 & - \\
\hline $8-$ HMS & AVC & 30 & 42 & 19 & 2.27 & 1.00 & 0.56 & 0.19 \\
\hline $9-\mathrm{APM}$ & AVC & 34 & 42 & 35 & 2.81 & - & 0.49 & 0.25 \\
\hline $10-E C F$ & CIV & 16 & 38 & 0 & 5.00 & 0.67 & 0.44 & 0.08 \\
\hline 11 - AAS & CIV & 16 & 24 & 29 & 1.60 & - & 0.86 & 0.22 \\
\hline 12 - FAM & AVC & 17 & 38 & 14 & - & - & 0.92 & 0.56 \\
\hline $13-\mathrm{CSM}$ & TGVB & 14 & 27 & 28 & - & 1.4 & 0.80 & 0.59 \\
\hline \multicolumn{2}{|c|}{ MÉDIA } & 21 & 38 & $20(p<0.01)$ & 2.7 & $0.92(p<0.01)$ & 0.73 & $0.28(p<0.01)$ \\
\hline
\end{tabular}

ABREVIATURAS - Ident - identificação, Diag - diagnóstico, T.P.O. - tempo de posoperatório, Def. Pond. - déficit ponderal, FP/FS - fluxo pulmonar - fluxo sistêmico, PSAP/PSAo - pressão sistólica em artéria pulmonar — pressão sistólica na aorta.

três dias, quando apresentou bloqueio atrioventricular total. $O$ outro paciente faleceu no vigésimo dia de pós-operatório, em septicemia. Os demais pacientes evoluíram, satisfatoriamente, recebendo alta hospitalar em média duas semanas de pós-operatório. Dois pacientes faleceram na evolução tardia em broncopneumonia.

Trinta e seis pacientes foram acompanhados, tendo boa evolução, com controle satisfatório da insuficiência cardíaca.

A média pós-operatória do peso corpóreo dos pacientes foi de 7,5 quilogramas $(4,5$ a 10,9$)$, tendo apresentado um ganho mensal médio de 400 gramas, sensivelmente superior à dos pacientes com shunt não tratados cirurgicamente ${ }^{6}$.

Treze pacientes foram estudados, hemodinamicamente (Figuras 1 e 2 e Tabela 1 ).

Nestes casos, o déficit ponderal médio de 37 por cento foi reduzido para 21 por cento no pós-operatório, num tempo médio de reestudo de 20 meses $(p<0,01)$.

A média das relações da pressão arterial pulmonar - pressão arterial sistêmica, no pré-operatório foi de 0,73 e de 0,28 no pós-operatório ( $p$ $<0,01)$.

A média das relaçōes fluxo pulmonar/fluxo sistêmico no pré-operatório foi de 2,7 , reduzindo-se para 0,92 no pós-operatório $(p<0,01)$.
A análise separada dos 6 casos de atrioventricularis communis mostra uma mortalidade hospitalar de 16,7 por cento ( 1 caso) e os 5 sobreviventes tiveram evolução clínica bastante satisfatória, com diminuição do déficit ponderal de 38 para 23 por cento. Os cinco pacientes sobreviventes foram reestudados, hemodinamicamente, em média 22 meses após a cerclagem. A relação pressão arterial pulmonar - pressão arterial sistêmica caiu de 0,69 para 0,31. A média das relações entre fluxo pulmonar - fluxo sistêmico caiu de 2,7 para 0,92 no pós-operatório.

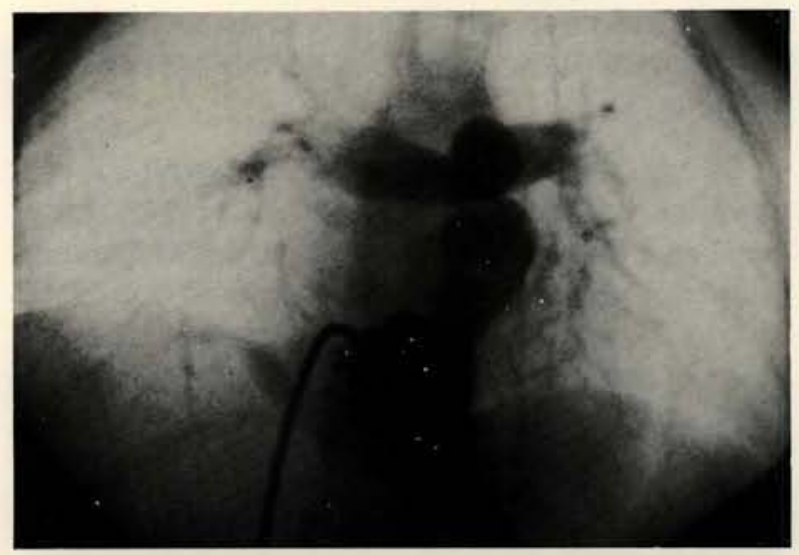

Fig. 1 - Cineangiografia pós-operatoria mostrando a artéria pulmonar cerclada (póstero-anterior). 
GREGORI Jr., F.; TAKEDA, R.; FAÇANHA, L.; RIBEIRO. I.; FABIANI, M.; GALVĀO, G.; ROCHA, F. G.; GÓIS, L. E.; AQUINO. W.: SIQUEIRA, J. E.; KRELING, P.; MIKITA, L. C. - Dúvidas na indicação cirúrgica da cerclagem do tronco pulmonar. Rev. Bras. Cir. Cardiovasc., 1(2):1-8, 1986

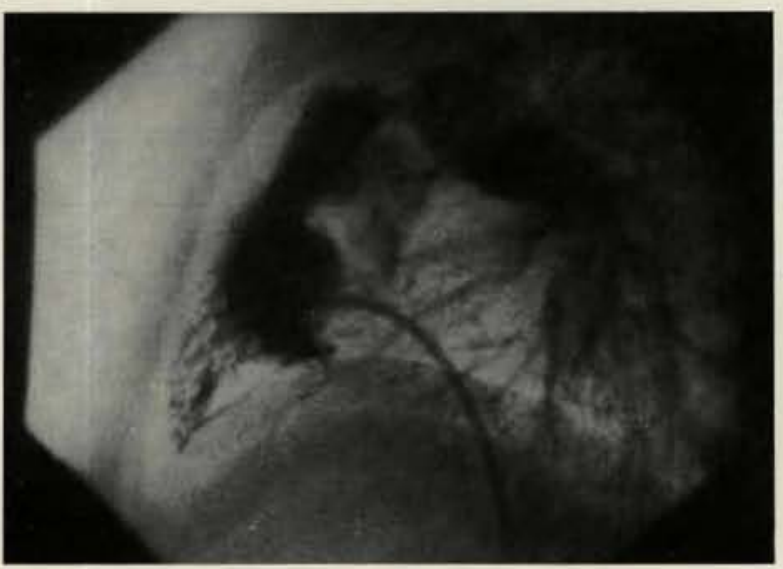

Fig. 2 - Cineangiografia pós-operatória de cerclagem a artéria pulmonar com o paciente em perfil

\section{COMENTÁRIOS}

A cerclagem do tronco pulmonar tem sido empregada como procedimento cirúrgico paliativo em diversas anomalias cardíacas congênitas. Devido aos resultados pós-operatórios contraditórios com este procedimento e ao grande desenvolvimento das técnicas cirúrgicas corretivas em crianças de baixo peso corpóreo e, principalmente, da circulação extracorpórea com hipotermia profunda, a cerclagem foi largamente abandonada, em favor das cirurgias corretivas.

O método tem a finalidade de, reduzindo-se o diâmetro do tronco pulmonar, haver uma diminuição do fluxo pulmonar, com conseqüente baixa da pressão arterial pulmonar, diminuindo-se, também, o retorno venoso ao coração esquerdo, com conseqüente diminuiçāo da sobrecarga ventricular e alívio da insuficiência cardíaca. A longo prazo, haveria, também, uma prevenção da doença vascular pulmonar.

Para uma cerciagem efetiva, baseamo-nos nas pesquisas de TRUSLER \& MUSTARD ${ }^{\beta}$ (1972), calculando-se o perímetro da fita em torno de $20 \mathrm{~mm}$ mais $1 \mathrm{~mm}$ por quilograma de peso corpóreo nos casos de comunicação interventricular simples. Pacientes com transposição das grandes artérias teriam uma resposta insatisfatória com esta fórmula, evoluindo, em geral, com cianose e bradicardia, havendo necessidade de desfazer-se a cerclagem. Medida aparentemente efetiva seria a de calcular-se o perímetro na base de $24 \mathrm{~mm}$ mais $1 \mathrm{~mm}$ por quilograma de peso corpóreo. Um simples milímetro de varia- ção da fita pode resultar num fluxo sangüineo pulmonar diferente, com cianose e/ou insuficiência cardíaca.

Nos casos de cardiopatias complexas com fluxo bidirecional, este princípio não é tão rígido, havendo necessidade de variaçōes na circunferência, em cada caso individual.

Tentando tornar mais objetivo o cálculo dà reduçāo do fluxo pulmonar, fazemos a medição simultânea da pressão hidrostática em tronco pulmonar antes e após a cerclagem, de tal forma a reduzi-la de 50 a 60 por cento nos acianóticos e de 40 por cento nos cianóticos, partindo sempre das medidas de circunferência descritas acima.

Observamos, muito comumente, que pacientes com a mesma malformação apresentaram respostas diferentes ao mesmo nível de cerclagem e atribuímos estas variações ao estado da rede vascular arterial pulmonar, à própria espessura da parede arterial pulmonar, ao grau de shunt bidirecional e à resposta contrátil da musculatura ventricular. Daí utilizarmos as medidas pressóricas, observando-se uma evolução pós-operatória, tanto imediata quanto tardia, bastante satisfatória. Houve uma prevenção do desenvolvimento de doença vascular pulmonar e importante melhora do quadro da insuficiência cardiaca, nos nossos casos. Tão importante quanto isto foi a baixa mortalidade hospitalar observada em nossa casuística (5 por cento), quando comparada aos resultados da literatura. ALBUS et alii ${ }^{1}$ (1984), numa análise de 182 casos do Hospital for Sick Children de Toronto, Canadá, subdividem a casuística em três subgrupos: o primeiro grupo de pacientes com defeitos tipo comunicação interventricular, com 9 por cento de óbitos imediatos; o segundo grupo com transposição das grandes artérias, com 13,5 por cento e o terceiro, cardiopatias complexas com mortalidade de 48 por cento, de tal forma que, se analisarmos globalmente a casuística, a mortalidade hospitalar ficaria em torno de 16 por cento.

Avaliando-se os nosso dois óbitos, um ocorreu por septicemia no vigésimo dia de pós-operatório e não podemos atribuir a falha técnica na cerclagem da artéria pulmonar; o outro, tratava-se de paciente com 20 dias de idade, 1,7 quilograma de peso corpóreo e com diagnóstico de atrioventricularis communis totalis; desenvolveu um bloqueio atrioventricular total, falecendo após três dias de excelente evolução pós-operatória.

Os demais pacientes com AVCT evoluiram muito bem, tanto no pós-operatório imediato, quan- 
GREGORI Jr., F.; TAKEDA, R.; FAÇANHA, L.; RIBEIRO, I.; FABIANI, M.; GALVÃO, G.; ROCHA, F. G.; GÓIS, L. E.; AQUINO, W.: SIQUEIRA, J. E.; KRELING, P.; MIKITA, L. C. - Dúvidas na indicação cirúrgica da cerclagem do tronco pulmonar. Rev. Bras. Cir. Cardiovasc., 1(2):1-8, 1986.

to tardio, tendo sido reoperados para correção total, com boa evolução. É polêmica a indicação ou não da cerclagem, nestes casos. EPSTEIN et alii ${ }^{2}$ (1979) concluem ser a cerclagem um procedimento cirúrgico efetivo nos pacientes com AVCT com predomínio de shunt intracavitário e insuficiência mitral mínima. KIRKLIN \& BLACKSTONE ${ }^{3}$ (1979), em editorial, contestam os resultados do EPSTEIN et alii ${ }^{2}$, principalmente devido ao pequeno número de casos e sugerindo tratar-se a casuística de um grupo selecionado de pacientes. Preferem, portanto, a cirurgia corretiva como primeiro procedimento.

A mesma crítica poderia ser feita nos nossos 6 casos de AVC total, por se tratar de um número pequeno de casos, apesar de o comportamento pós-operatório tardio tem sido similar ao dos pacientes com comunicação interventricular isolada.
Portanto, considerando a multiplicidade das técnicas utilizadas para a realização da cerclagem, podemos deduzir daí os diversos resultados observados na literatura mundiall ${ }^{1,2,3,4,7}$.

Assim, observamos, no nosso estudo, uma baixa mortalidade pós-operatória $(5 \%)$ e uma meIhora significativa, tanto clínica como laboratorial, incluindo os casos com AVCT.

Continuamos, portanto, a empregar a cerclagem do tronco pulmonar como primeiro procedimento cirúrgico nos pacientes com cardiopatias congênitas com hiperfluxo pulmonar, hipodesenvolvimento ponderal e insuficiência cardiaca rebelde a tratamento clínico.

GREGORI Jr., F.; TAKEDA, R.; SILVA, S. S.; FAÇANHA, L.; RIBEIRO, I.; FABIANI, M.; GALVĀO, G.; ROCHA, F. G.; GÓIS, L. E.; AQUINO, W.; SIQUEIRA, J. E.; KRELING, P.; MESQUITA, L. C. - Questions regarding surgical indications in pulmonary artery banding. Rev. Bras. Cir. Cardiovasc., 1 (2):1-8, 1986.

ABSTRACT: From February 1979 to March 1986, 50 patients were submitted to pulmonary artery banding in order to control congestive heart failure and to prevent pulmonary vascular disease. Twenty-three patients were male and 17 were female. The mean age was 6 months ( 20 days to 17 months). The preoperative diagnosis were: ventricular septal defect (22), atrioventricular canal (6), double outlet right ventricle (2), tricuspid atresia (2), transposition of the great arteries (3), truncus arteriosus (2), single ventricle (1) and complexes pathologies (2). The pulmonary banding was carried out under control of the pulmonary artery pressure. Two patients ( 5 per cent) died on the immediate postoperative period: one patient in atrioventricular block and another in sepsis. One patient died of pneumonia on the late postoperative period. The remaining patients had a satisfactory evolution, increasing $400 \mathrm{~g}$ of weight monthly. Thirteen patients were submitted to hemodynamic studies on the postoperative period (mean 21 months). Important reduction in pulmonary pressures and flows were then observed, including in five cases with A-V canal. Thirteen patients were reoperated on to correct their underlying defects one year after the banding. Then, one patient died in the immediate postoperative phase and another on the late phase. One of the six patients with A-V canal died on the immediate postoperative period and the others were reoperated for correction of the defects, with good results.

DESCRIPTORS: pulmonary artery banding.

\section{REFERÊNCIAS BIBLIOGRÁFICAS}

1 ALBUS, R. A.; TRUSLER, G. A.; IZUKAWA, T.; WILLIAMS, W. G. - Pulmonary artery banding. J. Thorac. Cardiovasc. Surg., 88 (5): 645-653, 1984.

2 EPSTEIN, M. L.; MOLLER, J. H.; AMPLATZ, K.; NICOLOFF, D. M. - Pulmonary artery banding in infants with complete atrioventricular canal. J. Thorac. Cardiovasc. Surg., 78 (1): 28-31, 1979.

3 KIRKLIN, J. W. \& BLACKSTONE, E. H. - Management of the infant with complete atrioventricular canal. $J$. Thorac. Cardiovasc. Surg., 78 (1): 32-34, 1972.
4 MENAHEM, S. \& VENABLES, A. W. - Pulmonary artery banding in isolated or complicated ventricular septal defects: results and effects on growth. Br. Heart J., 34 (1): 87-94, 1972.

5 MULLER Jr., W. H. \& DAMMANN Jr., J. F. - The treatment of certain congenital malformations of the heart by the creation of pulmonic stenosis to reduce pulmonary hipertension and excessive pulmonary blood flow: a preliminary report. Surg. Gynecol. Obstet., 95 (1): 213:219, 1952. 
GREGORI Jr., F.; TAKEDA, R.; FAÇANHA, L.; RIBEIRO, I.; FABIANI, M.; GALVÃO, G.; ROCHA, F. G.; GOIS, L. E.; AQUINO, W.: SIQUEIRA, J. E.; KRELING, P.; MIKITA, L. C. - Dúvidas na indicação cirúrgica da cerclagem do tronco pulmonar. Rev. Bras. Cir. Cardiovasc., 1(2):1-8, 1986.

6 SILVA, S. S. - Influência das repercussōes hemodinâmicas de cardiopatias congênitas acianóticas sobre o crescimento. Rio de Janeigro, 1980. (Tese de Mestrado Pontifícia Universidade Católica do Rio de Janeiro).

7 STEWART, S.; HARRIS, P.; MANNING, J. - Pulmonary artery banding: an analysis of current risks, results, and indications. J. Thorac. Cardiovasc. Surg., 80 (3): 431-436, 1980.

8 TRUSLER, G. A. \& MUSTARD, W. T. - A method of banding the pulmonary artery for large isolated ventricular septal defect without transposition of the great arteries. Ann. Thorac. Surg., 13 (4): 351-355, 1972.

\section{Discussão}

DR. CARLOS R. MORAES

Recife, PE

Inicialmente, gostaria de me congratular com o Dr. Gregori e colaboradores, pelo trabalho apresentado, no qual relatam uma série consecutiva de 40 crianças submetidas a cerclagem de artéria pulmonar.

Não pretendo, nestes comentários, fazer uma apresentação paralela de casuística própria, mas apenas tirar algumas dúvidas que me ficaram, ao ler o resumo do trabalho. Aliás, nota-se, logo no título do trabalho, que os autores têm, ou tinham, dúvidas na indicação desta operação.

Todos nós sabemos que a tendência atual é se corrigirem as cardiopatias congênitas, sempre que possível, precocemente, sobretudo tratando-se de comunicação interventricular. Entretanto, todos também concordamos em que algumas crianças continuam a ter indicação para cerclagem. Penso, como os autores, que o grande problema da cerclagem é justamente a indicação. Porisso, gostaria de saber, pois, para mim, não ficou claro, quais os critérios adotados pelos autores para indicar a cerclagem. Basearam-se apenas na idade, peso e presença de insuficiência cardiaca, ou outros fatores, tais como intratabilidade da insuficiência cardia$\mathrm{ca}$, infecção pulmonar ativa, acidose metabólica e necessidade de suporte respiratório estavam presentes, neste grupo de doentes? Esse é um dado muito importante para que se possa comparar os resultados apresentados com outras séries. Com relação à técnica de cerclagem, gostaria de saber se os autores monitorizam apenas a pressāo em artéria pulmonar, ou se medem, também, a pressão em VD, para verificar qual o gradiente criado, e, simultaneamente, medem a pressão arterial sistêmica. Também gostaria de saber se avaliam a saturação arterial de oxigênio antes e após a cerclagem. Finalmente, um dado importante para mim é a avaliação do refluxo valvar no atrioventricularis, antes de se indicar a cerclagem. Os autores afirmam que predominava o shunt e não o refluxo valvar, entendendo-se, daí, que ele existia, embora pouco significativo. Minha pergunta é: como avaliam o refluxo valvar no atrioventricularis e como o classificam, no sentido de indicar ou não a cerclagem. Estas dúvidas me surgiram, ao ler o resumo do trabalho, e não foram esclarecidas na apresentação, mas, evidentemente, nāo tiram o mérito do mesma, que é muito bom. Parabéns.

\section{DR. MILTON A. MEIER}

Rio de Janeiro, RJ

Cumprimento ao Dr. Gregori, pela sua excelente apresentação e concordo com ele, plenamente, ao afirmar que ainda existem muitas dúvidas quanto à indicação precisa da bandagem da artéria pulmonar (AP). Apesar das teóricas vantagens hemodinâmicas da restrição do fluxo da $A P$, essa operação tem sido uma das mais controvertidas da cirurgia cardiaca. Quando Muller e Dammann propuzeram essa técnica, em 1963, o fechamento primário dos defeitos do septo ventricular em crianças de baixos peso e idade era acompanhado de mortalidade elevada. A bandagem, então, permitia que a operação definitiva fosse adiada e o paciente atingisse uma idade e peso, com o que a correção podia ser feita com mais segurança. A evolução da circulação extracorpórea e dos cuidados pré e pós-operatórios permitem, hoje, o fechamento primário dos defeitos do septo ventricular, mesmo em crianças nos primeiros meses de vida, com morbidade e mortalidade muito baixas. Várias razões devem ser levadas em consideração para uma visão crítica da bandagem em AP: 1) A mortalidade da operação é significativa (em torno de 16\%). A segunda operação, para a debandagem, também é acompanhada de mortalidade (10\%). Além disso, existe uma mortalidade pouco mencionada, que é a que pode ocorrer antes da segunda operação; 2) As complicações da operação são freqüentes, como: reoperação imediata para modificar a intensidade da restrição, estenose infundibular, migração da bandagem para a bifurcação da AP e, menos freqüentemente, estenose subaórtica. Depois da debandagem, pode ocorrer, ainda, a reestenose pulmonar. Apesar de todos esses inconvenientes, em alguns pacientes e em algumas lesōes anatômicas, a operação pode e deve ser indicada. Em pacientes com defeitos do septo ventricular, nos primeiros meses de vida, em mau estado geral, indicamos a bandagem da AP. Essa operação é, também, indicada em lactentes e mesmo em crian- 
GREGORI Jr., F.; TAKEDA, R.; FAÇANHA, L.; RIBEIRO, I.; FABIANI, M.; GALVÃO, G.; ROCHA, F. G.; GOIS, L. E.; AQUINO. W.; SIQUEIRA, J. E.; KRELING, P.; MIKITA, L, C. - Dúvidas na indicaçâo cirúrgica da cerclagem do tronco pulmonar. Rev. Bras. Cir. Cardiovasc., 1(2):1-8, 1986.

ças até 2 anos de idade, com defeitos septais múltiplos (tipo swiss-cheese), e defeitos septais associados à coarctação da aorta, de difícil manuseio clínico. Outras lesões anatômicas também podem se beneficiar com a bandagem da AP: 1) Nos defeitos do septo atrioventricular (atrioventricularis communis), em que a alteração hemodinâmica principal é o shunt esquerdo-direito com hiperfluxo pulmonar sem insuficiência importante da valva atrioventricular esquerda; 2) Nos casos de atresia tricúspide com hiperfluxo arterial pulmonar, em crianças ainda sem indicação para a operação de Fontan-Kreutzer; 3) Pacientes com truncus arteriosus com hiperfluxo arterial pulmonar e sem insuficiência da valva truncal; 4) Em várias malformaçōes complexas com hiperfluxo arterial pulmonar sem indicação para a correção completa, nas quais o tratamento clínico é difícil. Quanto à maneira de fazer a operação, tenho a certeza de que ela deve ser a mais simples e a mais fácil de ser reproduzida. A técnica descrita por Trusler é a de marcar uma fita de teflon com $20 \mathrm{~mm}$ somados ao peso da criança em milímetros. Outra técnica muito usada é a descrita por Brown, na qual a AP é amarrada com uma fita sobre uma vela de Hegar número 6 ou 7 . Se existe a possibilidade de medidas de pressâo confiáveis, a constricção da artéria pulmonar deve ser aumentada até se obter um gradiente entre o ventrículo direito e a AP de $25-50 \mathrm{mmHg}$, com o cuidado de manter a pressão de VD abaixo da pressão sistêmica. Uma vez conseguida a pressão desejada, o coração deve ser observado durante alguns minutos. Se ocorrerem bradicardia, arritmia grave, ou então a cor do músculo cardíaco se tornar muito escura, a bandagem deve ser avaliada. Agradeço aos organizadores deste congresso, pela oportunidade de comentar o excelente trabalho do Dr. Gregory e seu grupo.

DR. E. REGIS JUCÁ

Fortaleza, CE

Gostaria de cumprimentar o Dr. Gregory e seu grupo de Londrina por trazerem a este Congresso uma importante contribuição a um tema tão controvertido ainda hoje como é a bandagem da artéria pulmonar (B.A.P.). Até 1981, a operação paliativa de menos simpática indicação era a B.A.P., pelos resultados incertos e pelo alto gráu de subjetividade do procedimento. Em outras palavras: o quanto apertar? Após lermos o trabalho de BROWN e KING, da Universidade de Indiana, publicado no Surgical Clinics of North America, outubro 1981 , passamos a realizar a B.A.P. com ótimos resulta- dos em cardiopatias complexas de hiperfluxo. Estes autores aconselham apertar a fita da bandagem em cima de uma vela de Hegar n: 6, em pacientes até 3 meses de idade. Ao retirarmos a vela de Hegar, o orificio da pulmonar será de $28 \mathrm{~mm}^{2}$. Nós temos usado, em crianças maiores (com ventrículo único, por exemplo), a Hegar n: 7, que produz um orifício de $38 \mathrm{~mm}^{2}$ (Quadro). Em 1985, realizamos 6 bandagens da A.P., em cardiopatias complexas, sem óbito. Para terminar, devo dizer que a palavra cerclagem, de uso corrente em vários campos, não existe no dicionário do Aurélio Buarque de Holanda e sim a palavra BANDAGEM (Aurélio Buarque de Holanda, Nova Fronteira p. 181 - Edição 1975: "Bandagens: ato de bandar; faixa, atadura, ligadura. Do francês bandage".

Reiterando os meus cumprimentos ao Dr. Gregory, pergunto se a medição direta da pressão da artéria pulmonar em recém-nascidos tem sido fácil, confiável e sem morbidade. Agradeço o previlégio de comentar este trabalho.

\section{BANDAGEM DA ARTERIA PULMONAR}

1) ATÉ 3 MESES: HEGAR $6 \mathrm{~mm}$ Orificio: $28 \mathrm{~mm}^{2}$

(D $\left.=6 \mathrm{~mm}-\mathrm{r}=3 \mathrm{~mm} \mu \mathrm{r}^{2}=3.14 \times 3^{2}=28.26 \mathrm{~mm}^{2}\right)$.

2) 3 MESES a 1 ANO: HEGAR $7 \mathrm{~mm}$ ORIFICIO: $38 \mathrm{~mm}^{2}$

$\left(D=7 \mathrm{~mm}-\mathrm{r}: 3.5 \mathrm{~mm} \mu \mathrm{r}^{2}=3.14 \times 3.5^{2}=38.46 \mathrm{~mm}^{2}\right)$ Modificado de BROWN \& KING - Surg. Clin. North Am. Out. 1981

\section{DR. GREGORI}

(Encerrando)

Primeiramente, gostaria de agradecer os comentários elogiosos dos professores Carlos R. Moraes, Milton A. Meier e Regis Jucá. Após estes resultados muito felizes de nossa casuística, tem sido, para nós, mais fácil indicar a cerclagem da artéria pulmonar para os pacientes com cardiopatia congênita com hiperfluxo pulmonar. Estes baseiam-se, fundamentalmente, na insuficiência cardiaca intratável clinicamente e no hipodesenvolvimento pônderoestatural em pacientes com idade inferior a $6 \mathrm{me}$ ses, com patologia tipo comunicação interventricular e, evidentemente, com idade superior nas cardiopatias complexas. No início de nossa experiência, confesso, a indicação deste tipo de operação foi mais uma atitude de defesa, uma vez que engatinhávamos na cirurgia de crianças com baixo peso corpóreo, sendo os resultados pouco animadores. Com a melhoria dos resultados, passamos a questionar a indicação de cerclagem, porém a baixa mortalidade com a cirurgia paliativa e o excelente resultado pós-operatório continuaram a nos impelir para este tipo de operação como primeiro procedimento. A indicação passou a se estender para os casos de AVC total com pouco refluxo transvalvar e os mesmos resultados foram compa- 
GREGORI Jr., F.: TAKEDA, R.: FACANHA, L.: RIBEIRO, 1.; FABIANI, M.; GALVÃO, G.: ROCHA, F. G.; GÓIS, L. E.; AQUINO. W.: SIQUEIRA, J. E. KRELING, P.: MIKITA, L. C. - Dúvidas na indicação cirùrgica da cerclagem do tronco pulmonar. Rev. Bras. Cir. Cardiovasc., 1(2):1-8, 1986.

ráveis aos da CIV simples. Reconhecemos ser dificil o cálculo do refluxo transvalvar, tendo sido avaliado, em nosso Serviço, apenas pela cineventriculografia. Esta indicaçāo, diga-se de passagem, é bastante criticada pela literatura mundial. Achamos que a cerclagem da artéria pulmonar tem sido pou$c o$ indicada atualmente, não somente pelo avanço nas técnicas corretivas, mas também pelos maus resultados obtidos com a cirurgia paliativa, chegando, muitos cirurgiōes, a simplesmente nunca realizar este procedimento. Perguntamos: o que fazer com um recém-nascido com $2 \mathrm{~kg}$ de peso corpóreo, em franca insuficiência cardiaca; realizar a cerclagem, ou aguardar a evoluçåo natural com o tratamento clínico? Comparativamente, acreditamos que os melhores resultados obtidos, em nossa série, deveram-se, fundamentalmente, à técnica operatória empregada, visto que a cirurgia foi indicada em pacientes com baixo peso corpóreo, na grande maioria dos casos, sendo a média prejudicada pelos pacientes com cardiopatias complexas com peso corpóreo maior. Achamos que a medição das pressōes da artéria pulmonar, antes e após a cerclagem, de fundamental importância para um resultado efetivo. Por exemplo, usamos, algumas vezes, vela de Hegar e, após a constriç̧ão, não observamos redução da pressāo arterial pulmonar, sem, portanto, redução do fluxo arterial pulmonar. $O$ cálculo teórico do perímetro da fita pode ser utilizado de vários modos; porém, a comprovação da efetividade da cerclagem somente será comprovada pela redução efetiva do fluxo pulmonar traduzida pela diminuição da pressão, em nossa técnica, muito fácil de ser realizada, inclusive, em recém-nascidos. Não utilizamos a redução da pressão em ventrículo direito para o cálculo do gradiente transcerclagem, por não achá-la necessária; interessa-nos apenas a redução do fluxo pulmonar ao redor dos $50 \%$, diminuindo, assim, importantemente, o retorno sangüíneo ao coração esquerdo, ao mesmo tempo em que protegemos a rede arteriolar pulmonar. Finalizando, agradeço à Comissão Organizadora do Congresso Nacional de Cirurgia Cardíaca, pela seleção do nosso trabalho. 